\title{
Essais
}

ESSAIS

Revue interdisciplinaire d'Humanités

Hors-série 1 | 2013

L'estrangement

\section{Nos mots et les leurs. Une réflexion sur le métier de l'historien, aujourd'hui}

\section{Carlo Ginzburg}

Traducteur : Martin Rueff

\section{OpenEdition \\ Journals}

Édition électronique

URL : http://journals.openedition.org/essais/2527

DOI : $10.4000 /$ essais. 2527

ISSN : 2276-0970

\section{Éditeur}

École doctorale Montaigne Humanités

\section{Édition imprimée}

Date de publication : 15 octobre 2013

Pagination : 191-210

ISBN : 978-2-9544269-1-4

ISSN : $2417-4211$

\section{Référence électronique}

Carlo Ginzburg, « Nos mots et les leurs. Une réflexion sur le métier de l'historien, aujourd'hui », Essais [En ligne], Hors-série 1 | 2013, mis en ligne le 05 octobre 2020, consulté le 13 octobre 2020. URL : http://journals.openedition.org/essais/2527 ; DOI : https://doi.org/10.4000/essais.2527 


\section{Nos mots et les leurs. Une réflexion sur le métier de l'historien, aujourd'hui}

"C'est que la chimie avait le grand avantage de s'adresser à des réalités incapables, par nature, de se nommer elles-mêmes."

Marc Bloch

1. Dans ses réflexions de méthode publiées posthumes sous le titre Apologie pour l'histoire ou Métier d'historien Marc Bloch fait remarquer que : " [Car,] au grand désespoir des historiens, les hommes n'ont pas coutume, chaque fois qu'ils changent de mœurs, de changer de vocabulaire $»^{1}$.

Le résultat de cette divergence est l'ambiguïté sémantique. Considérons un mot fondamental de notre vocabulaire intellectuel et émotionnel - «liberté »dont les significations multiples ont été longtemps au cœur des préoccupations de Bloch. À les regarder de près on pourra jeter quelque lumière sur l'allusion ironiquement emphatique au " désespoir » des historiens quand ils se trouvent confrontés au fossé qui sépare la permanence des mots de leur signification changeante. Bloch parlait des " historiens " en pensant à lui-même : mais ses réactions personnelles avaient des racines à la fois plus lointaines et plus complexes.

1 J'ai présenté différentes versions de ce texte à Rome (Università della Sapienza), Be’er Sheva (Ben-Gurion University), Los Angeles (Department of History, UCLA), Berlin (Freie Universität). Je tiens à exprimer ma gratitude à Andrea Ginzburg, Christopher Ligota, Perry Anderson et (surtout) à Simona Cerutti pour leurs critiques, ainsi qu’à Sam Gilbert et Henry Monaco pour leur révision de la version anglaise. Mon ami Martin Rueff a traduit mon texte avec sa compétence et générosité habituelles.

Marc Bloch, Apologie pour l'histoire ou métier d'historien, in id., L'Histoire, la Guerre, la Résistance, édition établie par Annette Becker et Étienne Bloch, Paris, Gallimard, 2006, p. 872. Je dois à F. Ciafaloni d'avoir attiré une nouvelle fois mon attention sur ce passage : «Le domande di Vittorio. Un ricordo di Vittorio Foa ", Una città, n 176, luglio-agosto 2010, p. 42. 
2. "Histoire ", du grec historia, appartient aussi à ces mots de notre vocabulaire qui, traduit en plusieurs langues, est resté le même pendant vingtcinq siècles mais a changé de signification ${ }^{2}$. Après avoir été utilisé par les physiciens et les anatomistes, les botanistes et les antiquaires dans un sens qui incluait à la fois la "description » et "l'enquête ", histoire a fini par désigner presque exclusivement le royaume des actions humaines - même si des traces de son ancien usage peuvent être détectées dans des expressions telles que l'« histoire clinique » d'un patient. Le rétrécissement de sa signification peut être considéré comme un effet collatéral d'un tournant qu'on peut identifier, symboliquement, avec le fameux passage du Saggiatore de Galilée :

La philosophie est écrite dans ce vaste livre qui se trouve sans cesse ouvert sous nos yeux (je veux dire l'univers). Mais il ne peut être compris à moins qu'on n'ait d'abord appris à comprendre la langue et à reconnaître les caractères dans lesquels il se trouve écrit. Il est écrit dans le langage des mathématiques, et les caractères sont les triangles, les cercles et d'autres figures géométriques. Sans un tel moyen, il nous est impossible à nous les humains d'en comprendre un seul mot... ${ }^{3}$.

En dépit de sa proximité avec des savants engagés dans une approche non mathématique de la nature, Galilée annonçait que le langage de la nature était - ou se trouvait sur le point de devenir - le langage des mathématiques ${ }^{4}$. Au contraire, le langage de l'histoire est, et a toujours été, d'Hérodote à nos jours, un langage humain : le langage de la vie de tous les jours, quand bien même il s'appuierait sur des statistiques et des diagrammes. Mais les sources sur lesquelles l'historien s'appuie sont elles aussi la plupart du temps écrites dans le langage de la vie de tous les jours.

Bloch a intensément réfléchi sur cette contiguïté et ses implications :

2 Gianna Pomata et Nancy G. Siraisi (éd.), Historia. Empiricism and Erudition in Early Modern Europe, Cambridge, Mass, Harvard Univesity Press, 2005.

3 Galileo Galilei, Il Saggiatore, édition de Libero Sosio, Milano : Feltrinelli, 1965, p. 264 : « La filosofia è scritta in questo grandissimo libro che continuamente ci sta aperto dinanzi agli occhi (io dico l'universo), ma non si può intendere se prima non s'impara a intender la lingua, e conoscere i caratteri ne' quali è scritto. Egli è scritto in lingua matematica, e i caratteri son triangoli, cerchi, ed altre figure geometriche, senza i quali mezzi è impossibile a intenderne umanamente parola...». Je développe ici l'interprétation de ce passage que j'avais avancée dans "Spie: radici di un paradigma indiziario ", in Miti emblemi spie. Morfologia e storia, Turin, Einaudi, 1986, p. 172-173 ; "Traces ", in Mythes emblèmes traces, Lagrasse, Verdier, 2010, p. 218-294 surtout p. 250-255.

4 David Freedberg, The Eye of the Lynx. Galileo, His Friends, and the Beginnings of Modern Natural History, Chicago, Chicago University Press, 2002.

5 Carlo Ginzburg, "Spuren einer Paradigmengabelung. Machiavelli, Galilei und die Zensur der Gegenreformation”, in Sybille Krämer, Werner Kogge et Gernot Grube (éd.), Spur. Spurenlesen als Orientierungstechnik und Wissenskunst, Frankfurt am Main, Suhrkamp Verlag, 2007, p. 257-280. 
«Son vocabulaire », pouvons-nous lire dans une autre section de ses réflexions posthumes, «l'histoire le reçoit donc, pour la plus grande part, de la matière même de son étude. Elle l'accepte, déjà fatigué et déformé par un long emploi ; ambigu d'ailleurs, souvent dès l'origine, comme tout système d'expressions qui n'est pas issu de l'effort concerté des techniciens $»^{6}$. Ainsi, les historiens se trouvent face à une alternative : ou bien ils font écho à la terminologie qui se trouve utilisée dans leurs sources, ou bien ils recourent à une terminologie qui leur est étrangère. Le premier choix, observe Bloch, ne mène nulle part : parfois c'est la permanence de mots intrinsèquement ambigus dissimule leur évolution sémantique ; parfois ce sont des significations similaires se trouvent dissimulées par la multiplicité des termes. Reste alors la seconde possibilité qui ne va pas sans risques : des termes comme " machinisme " par exemple, sensés " aider l'analyse ", finissent " par dispenser d'analyser ». Pire : ils « fomentent l'anachronisme : entre tous les péchés, au regard d'une science du temps, le plus impardonnable »" Seuls des échanges entre les chercheurs (des « ententes»), conclut Bloch, permettront de conduire à la construction d'un vocabulaire commun des sciences de l'homme ; mais l'invention de nouveaux mots est toujours préférable à la projection tacite de nouvelles significations au sein de termes d'usage commun ${ }^{8}$.

Ainsi, un vocabulaire rigoureux devrait permettre à l'histoire de pallier sa faiblesse intrinsèque - le langage quotidien qu'il partage avec la plupart de ses sources. La référence à la terminologie artificielle de la chimie, qui revient constamment dans les pages de Bloch, est assez explicite : rarement l'historien n'avait été aussi proche du positivisme. Mais Claude Bernard, dans son Introduction à la médecine expérimentale (1865), un des classiques du positivisme - auquel Bloch fait référence avec une pointe de désaccord - avait noté dans un paragraphe intitulé "la critique expérimentale doit porter sur les faits, non sur les mots " que l'ambiguïté ne manque pas de menacer aussi les langages conventionnels de la science :

Quand on crée un mot pour caractériser un phénomène, on s'entend en général à ce moment sur l'idée qu’on veut lui faire exprimer et sur la signification exacte qu'on lui donne, mais plus tard, par le progrès de la science, le sens du mot change pour les uns, tandis que pour les autres le mot reste dans le langage avec sa signification primitive. Il en résulte alors une discordance qui, souvent, est telle, que des hommes, en employant le même mot, expriment des idées très différentes. Notre langage n'est en effet qu'approximatif, et il est si peu précis, même dans les sciences, que, si l'on perd les phénomènes de vue, pour s'attacher aux mots, on est bien vite en dehors de la réalitée

6 Marc Bloch, Apologie pour l'histoire, p. 959.

7 Ibid., p. 969.

8 Ibid., p. 971.

9 Claude Bernard, Introduction à l'étude de la médecine expérimentale, Paris : J. B. Baillière et Fils, 1865, p. 330-331. Bloch renvoie à l'Introduction de Claude Bernard in Apologie pour l'histoire, p. $831,908$. 
3. Mais qu'en est-il, dans la perspective de l'historien, de la relation entre les mots - les mots qu'il trouve dans ses sources - et la réalité ? Plusieurs éléments s'entremêlent dans les réponses fournies par Bloch à une telle question. En premier lieu, un sens de l'inadéquation des mots face à ce qui les fait naître : passions, sentiments, pensées, besoins. Bloch illustre cette inadéquation en prenant un cas limite :

Quel enseignement si - le dieu fût-il d'hier ou d'aujourd'hui - nous réussissions à atteindre sur les lèvres des humbles leur véritable prière! À supposer, cependant, qu'ils aient su, eux-mêmes, traduire, sans le mutiler, les élans de leur cœur.

Car là est, en dernier ressort, le grand obstacle. Rien n'est plus difficile à un homme que de s'exprimer soi-même. (...) Les termes les plus usuels ne sont jamais que des approximations $)^{10}$.

De tels propos, qui plongent dans les recherches personnelles de Bloch, ne sont pas dictés par le scepticisme - loin s'en faut. La conscience de l'inadéquation de chaque mot, a suggéré à Bloch des stratégies indirectes qui lui ont permis de lire les sources médiévales en filigrane. Il suffit de se rappeler les pages magnifiques que Bloch consacre, dans Les rois thaumaturges aux hommes et aux femmes, atteintes de scrofules, qui traversaient d'énormes distances en espérant l'imposition miraculeuse de la main royale ${ }^{11}$. Mais c'est la même conscience qui l'a poussé à s'engager dans une histoire comparative, fondée, comme dans le cas de Les Rois thaumaturges, sur des catégories et des termes inévitablement éloignés de ceux utilisés dans les sources.

4. Ces éléments de réflexion ont pris le dessus dans un essai de 1928 intitulé " Pour une histoire comparée des sociétés européennes ». Il s'agit d'une espèce de manifeste méthodologique qui reste aujourd'hui encore un point de référence indispensable ${ }^{12}$. Dans la conclusion de son essai, Bloch évoque le préjugé tenace qui veut voir dans l'histoire comparative la recherche d'analogies, fussent-elles les plus superficielles. Or toute l'affaire de l'histoire comparative, insiste Bloch, est au contraire de souligner les différences spécifiques qui séparent les phénomènes dont elle s'occupe. C'est pourquoi il convient de rejeter toute fausse ressemblance : et par exemple, dans le domaine du Moyen Âge européen, la soi disant équivalence entre le villainage anglais et le servage français. Certes, des croisements sont indéniables :

10 Marc Bloch, Apologie pour l'histoire, p. 965.

11 Marc Bloch, Les rois thaumaturges, préface de Jacques Le Goff, Paris, Gallimard, 1983, p. 89-157.

12 Marc Bloch, "Pour une histoire comparée des sociétés européennes ", in CharlesEdmond Perrin (éd.), Mélanges historiques, I, Paris : SEVPEN, 1963, p. 16-40 ; in L'Histoire, la Guerre, la Résistance, op. cit., p. 349-380. 
Serf et villain sont tous les deux considérés, tant par les juristes que par l'opinion commune, comme privés de "liberté ", qualifiés à ce titre, dans certains textes latins, de servi [...] ; enfin, en vertu même de cette absence de "liberté " et de ce nom servile, volontiers assimilés, par les personnes savantes, aux esclaves romains.

Mais il s'agit, selon Bloch, d'une

analogie superficielle : le concept de non-liberté a beaucoup varié, dans son contenu, selon les milieux et les temps ${ }^{13}$.

Pour résumer : nous avons deux contextes géographiques différents, l'Angleterre et la France, et deux mots différents, villain et serf. Les juristes du Moyen Âge ainsi que les hommes cultivés les ont confondus avec servi, le terme qui désignait les esclaves à Rome parce que les mots de villain, de serf et de servi étaient employés pour désigner ceux qui ont été privés de liberté. Bloch refuse cette conclusion superficielle, sur la base d'un argument avancé par un grand nombre de chercheurs, au rang desquels Paul Vinogradoff, le grand médiéviste anglo-russe : à savoir qu'en 1300 environ, les villains avaient rejoint en Angleterre la catégorie des "free tenants "; en France pendant la même période les tenanciers étaient rigoureusement distingués des serfs. Bloch retrace ces différentes trajectoires historiques et conclut :

Le serf français du XIV siècle, le villain ou serf anglais du même temps ? Ce sont deux classes nettement dissemblables. Vaut-il la peine de les comparer? Assurément, mais afin, cette fois, de marquer leurs contrastes, par où s'exprime une opposition naissante entre le développement des deux nations ${ }^{14}$.

Ici, comme dans d'autres passages du même essai, Bloch utilise le mot classes pour identifier deux réalités sociales différentes confondues par erreur par les juristes médiévaux. Mais son commentaire des normes établies par les juristes anglais qui assignaient une moindre liberté à ceux qui devaient accomplir de lourdes corvées agricoles, suit une toute autre direction. «En établissant ces normes ", poursuit Bloch, "théoriciens et juges anglais n’inventaient rien. Ils ne faisaient que puiser dans un courant de représentations collectives, plus ou moins confusément élaborées, depuis longtemps, par les sociétés médiévales, celle du continent aussi bien que celle de l'île. L'idée que les travaux agricoles ont en eux-mêmes quelque chose d'incompatible avec la liberté répond à des vieux penchants de l'âme humaine; elle s'exprimait, à l'époque barbare, dans les mots d'opera servilia, fréquemment employés pour désigner ce genre d'ouvrages ${ }^{15}$. Bloch délaisse alors le terrain de la terminologie attestée par les documents et passe de manière abrupte à un terrain hypothétique, plus glissant : celui des "représentations collectives». Il emprunte cette notion

13 Marc Bloch, "Pour une histoire », p. 28 ; L'Histoire, la Guerre, la Résistance, op. cit., p. 364.

14 Marc Bloch, "Pour une histoire ", p. 30 ; L'Histoire, la Guerre, la Résistance, op. cit., p. 367.

15 Marc Bloch, "Pour une histoire », p. 31 ; L'Histoire, la Guerre, la Résistance, op. cit., p. 368. 
à Durkheim, dont le nom attire un éloge tout particulier en note. Dans un passage précédent, Bloch avait fait allusion à « un courant de représentations collectives, plus ou moins confusément élaborées depuis longtemps ${ }^{16}$.

La liberté et le servage au Moyen Âge, considérés dans une perspective plus ample, allaient refaire surface quelques années plus tard dans un autre essai de Bloch. Dans certains cas, les termes juridiques se référant à la liberté n'ont pas changé : mais leur signification (faisait remarquer Bloch) a subi des variations imperceptibles à travers le temps, comme l'attestent les documents carolingiens. Ils montrent une série de glissements, «évidemment inconscients " qui doivent être pris pour ce qu'ils sont : de la même manière les linguistes ont remarqué qu'à un certain moment de son histoire, le verbe labourer a pris la signification du mot latin $\operatorname{arare}^{17}$. Les historiens, écrit Bloch, devraient suivre l'exemple des linguistes et se garder de substituer leurs propres interprétations aux interprétations données dans le passé ${ }^{18}$.

Il s'agit d'une certaine manière d'un jugement inattendu. Dans un passage de son essai précédent Bloch avait rejeté l'assimilation de la servitude médiévale à l'ancien esclavage, inspiré par le mot latin servi. Et pourtant, on pourrait soutenir que reconstruire les perspectives des juristes et souligner leurs limites ne sont pas deux objectifs incompatibles. Mais il y a plus. L'essai dans lequel Bloch invite les historiens à prendre les linguistes comme modèles s'intitule "Liberté et servitude personnelles au Moyen âge, particulièrement en France : contribution à l'étude des classes» (1933). Pour Bloch, la catégorie moderne de " classe ", loin d'effacer les catégories avancées par les juristes médiévaux, permet de les inscrire dans une perspective qui est la nôtre et non pas la leur. Bloch le souligne dans les dernières pages de l'essai :

Ainsi nous nous trouvons ramenés de toutes parts à la même leçon. Les institutions humaines étant des réalités d'ordre psychologique, une classe n'existe jamais que par l'idée qu'on s'en fait. Écrire l'histoire de la condition servile, c'est, avant tout, retracer, dans la courbe complexe et changeante de son développement, l'histoire d'une notion collective : celle de la privation de liberté ${ }^{19}$.

Il est inutile de le dire, on peut accepter, discuter ou rejeter l'interprétation psychologique de "classe " mise en avant par Bloch en se fondant sur différentes catégories analytiques. Mais ses réflexions font naître une question plus générale : quelle est la relation entre les catégories de l'observateur et celles de l'acteur, telles qu'on peut les tirer des documents médiévaux ? Une autre

16 Marc Bloch, «Pour une histoire », p. 30 note 1, p. 29 note 2 ; L'Histoire, la Guerre, la Résistance, op. cit., p. 368 et p. 367 note 21 .

17 Marc Bloch, "Liberté et servitude personnelles au Moyen âge, particulièrement en France : contribution à l'étude des classes» [1933], Mélanges historiques, I, p. 286-355, surtout p. 332.

18 Marc Bloch, Mélanges historiques, I, p. 286-355, p. 327-328.

19 Marc Bloch, «Liberté et servitude », Mélanges historiques, I, p. 355. Cf. Carlo Ginzburg, «A proposito della raccolta dei saggi storici di Marc Bloch ", Studi medievali, s. 3, VI, 1965, p. 335-353. 
question s'ensuit immédiatement. Les juristes médiévaux étaient à la fois observateurs et acteurs. Quelle est la relation entre la représentation de la servitude partagée par les juristes et la représentation de la servitude partagée par les serfs ?

5. Une telle question, que Bloch n'a jamais posée de manière explicite, ne manque pas d'émerger de ses propres recherches. Je dois ici faire une digression de nature personnelle. C'est en lisant Les Rois thaumaturges en 1959, quand j'avais vingt ans, que je me suis décidé à essayer d'apprendre le métier d'historien. Quelques mois plus tard, j'ai décidé de m'engager dans l'étude des procès de sorcellerie, en me concentrant non pas sur la persécution en tant que telle mais sur les hommes et les femmes qui se présentaient devant les juges. Je devais cette impulsion à quelques livres (les Carnets de prison d'Antonio Gramsci, Le Christ sest arrêté à Eboli de Carlo Levi, Le monde magique d'Ernesto de Martino) ainsi qu'à des souvenirs poignants de la persécution raciale. Mais ce n'est que bien des années plus tard que j'ai pris conscience que mon expérience d'enfant juif pendant la guerre m'avait conduit à m’identifier avec les hommes et les femmes accusées de sorcellerie ${ }^{20}$.

Suivant le conseil de mon mentor Delio Cantimori, j'ai commencé à étudier les procès de l'Inquisition (un grand nombre concernaient en effet la sorcellerie ou des crimes qui s'y trouvaient reliés) conservés dans les Archives d'État de Modène. De là j'ai étendu ma recherche à d'autres archives - un voyage des plus erratiques puisque je n'avais aucun plan préétabli. Au début des années 1960, en parcourant les minutes des procès conservées dans les Archives d'État de Venise, je suis tombé sur un document dont j'ai tout de suite perçu qu'il représentait une anomalie complète : quelques pages, datées de 1591, rapportant la mise en examen de Menichino della Nota, un jeune bouvier du Frioul. Menichino répondait aux questions de l'inquisiteur en affirmant qu'il était un benandante. La signification de ce terme m'était inconnue - elle ne l'était pas moins pour l'inquisiteur qui semblait écouter avec stupeur l'histoire du prévenu. Comme il était né coiffé, disait Menichino, il était obligé de quitter son corps trois fois par an, " comme une fumée ", et de voyager avec les autres benandanti pour combattre " pour la foi contre les sorcières » sur la prairie de Josaphat ». "Lorsque les benandanti étaient vainqueurs ", concluait-il, "c était signe de bonnes récoltes ${ }^{21}$.

Il y a longtemps maintenant que j'ai proposé une analyse rétrospective de mes réactions face à ce document trouvé par hasard : le premier de la cinquantaine de procès que j'ai découverts par la suite dans les Archives Ecclésiastiques

20 Carlo Ginzburg, "Streghe e sciamani" [1993], in Il filo e le tracce. Vero falso finto, Milano: Feltrinelli, 2006, p. 281-293 ; "Sorcières et chamanes ", in Le Fil et les traces, Vrai Faux Fictif, Lagrasse, Verdier, 2010, p. 425-445.

21 Carlo Ginzburg. I benandanti, Turin 1966, p. 84-87; Les Batailles nocturnes, Paris, Flammarion, 1980, p. 130. 
d'Udine. Or tous ces procès tournent autour d'un mot - benandante - qui provoquait l'interrogation des inquisiteurs; les réponses des prévenus regorgeaient de détails extraordinaires. Les procès montrent que les inquisiteurs ne tardaient pas à prendre leur parti : les benandanti, qui prétendaient combattre contre les sorcières et les sorciers, n'étaient rien d'autre que des sorciers. De telles accusations provoquaient des rejets indignés de la part des benandanti, qui tenaient à décrire leur " profession " (c'est ainsi qu'ils l'appelaient) parfois avec fierté, parfois comme le résultat d'une impulsion obscure et inéluctable. Mais après cinquante ans d'enquêtes, ceux qui croyaient qu'ils avaient combattu du bon côté finirent par introjecter l'image hostile que les interrogateurs avaient construite. Tel fut le résultat d'un choc culturel imprégné de violence - dans ce cas, essentiellement symbolique. Le prestige des inquisiteurs, tout comme l'angoisse imminente de la torture et la mort sur l'échafaud avaient démontré leur pouvoir.

Dans un livre publié en 1966, traduit en français sous le titre Les Batailles nocturnes, j'ai analysé les contes fournis par les benandanti comme un fragment de culture paysanne que les stéréotypes de l'inquisition avaient progressivement déformé. Cet argument était basé sur les désaccords profonds qui opposaient les accusés et les inquisiteurs sur la signification réelle du terme benandante. Ce qui rendait l'extraordinaire dossier frioulan si important pour l'historien était l'absence presque totale de communication entre les deux parties engagées dans un débat dramatiquement inégal.

Après une pause qui dura plusieurs années je suis revenu vers mon travail sur les procès de sorcellerie. À ce moment j'ai compris que mon approche des juges, à la fois laïcs et ecclésiastiques, n'avait pas été adéquate pour plusieurs raisons. Les juges étaient souvent conduits par un véritable désir de trouver une signification aux croyances et aux actes des accusés - pour les éradiquer bien sûr. La distance culturelle pouvait engendrer un authentique effort de compréhension, de comparaison, de traduction. Permettez-moi de rappeler un cas limite mais fort éclairant. En 1453, l'évêque de Bressanone - le philosophe Nicolas de Cuse - écoute les récits racontés par deux vieilles femmes venues d'une vallée proche. Dans un sermon prononcé quelques temps après, il décrit ces femmes comme "à moitié folles " (semideliras). Elles ont rendu hommage à une déesse nocturne qu'elles appellent "Richella " (de "ricchezza ", qu'on peut traduire par "richesse »). L'évêque savant identifie Richella avec Diana, Abundia et Satia : les noms mentionnés dans les sections que les encyclopédies médiévales et les traités de droit canon consacraient aux superstitions populaires $^{22}$. Cet essai herméneutique était à certains égards exceptionnels. Et pourtant des juges et des inquisiteurs moins illustres ont dressé des sommaires

22 Carlo Ginzburg, Storia notturna. Una decifrazione del sabba, Turin, Einaudi, 1989, p. 70-73, 107-108 ; Le Sabbat des sorcières, Paris, Gallimard, 1992, p. 104-106, 135. 
et proposé des traductions qui créent des séries de boîtes chinoises imbriquées qui finissent par arriver jusqu'à l'interprète moderne - dans ce cas, moi-même. Je découvrais alors, non sans une certaine gêne, qu’à côté de mon identification avec les victimes, une contiguïté intellectuelle troublante me liait aux persécuteurs. J'ai essayé de rendre compte de cette condition dans un essai intitulé "L'inquisiteur comme anthropologue $»^{23}$.

6. Je ne saurais imaginer quelle direction auraient prise mes recherches - à commencer par celles que j'ai menées dans les archives du Frioul - si je n'avais jamais croisé les écrits de Bloch. Avec le recul j'aurais tendance à comparer les rêves extatiques aux "vraies prières" des humbles évoquées par Bloch : des expériences intérieures que les mots (attestés dans un cas, imaginés dans l'autre) ne peuvent enregistrer que de manière inadéquate. Dans le cas des benandanti, nous nous trouvons face à des mots utilisés par ordre de l'inquisiteur, puis transcrits par les notaires des inquisiteurs : un conflit contextuel (fût-il réglé par la loi) qu'il faut prendre en compte, quoiqu'il ne diminue en rien l'importance du témoignage.

Je suis enclin à croire qu'aucun historien n'aurait laissé passer un conflit aussi flagrant. Mais selon moi, la perception de ma contiguïté avec les inquisiteurs, dont je n'ai pris conscience que bien longtemps après, était nettement moins évidente. Peut-être la contiguïté ne s'est elle imposée à moi qu'après qu'avait grandi la conscience des racines profondes sous-jacentes aux choix préliminaires qui avaient modelé mon projet de recherche dès le départ.

L'identification émotionnelle avec les victimes, la contiguïté intellectuelle avec les inquisiteurs : nous voici bien loin des éléments qui, dans le modèle de la recherche historique décrite par Bloch, semblent le plus près du positivisme. Dans ses réflexions sur la nomenclature, le conflit apparaît seulement du côté de l'acteur : par exemple, dans ses remarques sur un phénomène comparativement tardif comme la conscience de classe, qu'il s'agisse des travailleurs du $\mathrm{XX}^{\mathrm{e}}$ siècle ou des paysans à la veille de la Révolution française ${ }^{24}$. Mais du côté du langage de l'historien-observateur, là où Bloch voulut se conformer, autant que faire se pouvait, au langage neutre et détaché des sciences naturelles, le conflit n'est jamais mentionné.

Dans la perspective que je préconise, une attitude critique et détachée peut être un but. Elle ne constitue jamais un point de départ. Bien que la finalité ne soit pas différente de celle de Bloch, les voies qui y mènent le sont. À la lumière de la contiguïté dangereuse entre le langage de l'historien et le langage

23 Carlo Ginzburg, "The Inquisitor as Anthropologist", in Clues, Myths, and the Historical Method, London and Baltimore, Johns Hopkins University Press, 1989, p. 156-164 ; "L'Inquisiteur comme anthropologue ", Le Fil et les traces, op. cit., p. 407-424.

24 Marc Bloch, Apologie, op. cit., p. 966. 
du témoignage, la stérilisation des instruments d'analyse est plus urgente que jamais - et d'autant plus dans des cas qui montrent une contiguïté entre l'observateur et les acteurs observateurs (l'inquisiteur comme anthropologue, l'inquisiteur comme historien).

7. Ces réflexions rétrospectives sur les recherches que j'ai pu mener dans les archives du Frioul dans les années 1960 et 1970 sont en partie inspirées par ma rencontre plus tardive avec les écrits de Kenneth L. Pike. Pike, le linguiste américain, anthropologue et missionnaire, a souligné l'opposition entre deux niveaux d'analyse, celui de l'observateur, et celui de l'acteur, baptisés respectivement niveau étique (de phonétique) et niveau émique (de phonémique). Partant du langage, Pike a fini par mettre sur pieds une théorie unifiée de la structure du comportement humain - le titre de son ouvre la plus ambitieuse, publiée d'abord en trois parties entre 1954 et 1960 pour être ensuite réimprimée dans une version révisée et étendue en 1967.

Le point de vue étique, explique Pike, permet d'examiner les langages et les cultures selon une perspective comparative ; le point de vue émique est " culturellement spécifique, appliqué à un seul langage ou à une seule culture à la fois $»^{25}$. Mais cette opposition statique et plutôt figée laisse vite la place à une version plus dynamique et réaliste :

Présentation préliminaire contre présentation finale : ainsi, les données étiques offrent l'accès au système - le point de départ de l'analyse. Elles offrent des résultats provisoires, des unités provisoires. L'analyse finale, ou la présentation, consisterait plutôt en unités émiques. Dans l'ensemble de l'analyse, la description étique initiale est progressivement raffinée et doit se trouver pour finir - en principe et sans doute jamais vraiment en pratique - remplacée par une description totalement émique ${ }^{26}$.

25 On trouve un écho de cette définition in Sanjay Subrahmanyam, "Monsieur Picart and the Gentiles of India ", in Lynn Hunt, Margaret Jacob et Wijnand Mijnhardt (éd.), Bernard Picart and the First Global Vision of Religion, Los Angeles, The Getty Research Institute, p. 197-214, surtout p. 206 (étique, c'est-à-dire. " universaliste " vs émique, "internaliste »).

26 Kenneth L. Pike, Language in Relation to a Unified Theory of the Structure of Human Behavior, $2^{\text {e }}$ édition révisée, The Hague, Mouton, p. 37-39. La dernière phrase de ce passage est citée par Marvin Harris qui la désapprouve in « History and Significance of the Emic/Etic Distinction ", Annual Review of Anthropology, 5, 1976, p. 329-350. L'article de Harris s'achève sur une critique de l'attitude de Claude Lévi-Strauss, qui se trouve qualifiée " d'osbcurantiste ». L'énorme tétralogie des Mythologiques (1964-1971) venait de paraitre et Lévi-Strauss y exprimait son dédain pour cette distinction, considérant la dimension «étique " comme nulle et non avenue, c'est-à-dire identifiant la véritable dimension " éthique " avec la dimension " émique " : "Structuralisme et écologie " [1972], in id., Le regard éloigné, Paris, Plon, 1983, p. 143-166, surtout, p. 161-162. On trouve à ce propos des remarques utiles in JeanPierre Olivier de Sardan, "Émique », L'Homme, 147, 1988, p. 151-166. Je tiens à remercier Simona Cerutti d'avoir attiré mon attention sur ce texte. Mon désaccord avec Harris et avec Lévi-Strauss (à un tout autre niveau bien évidemment) va émerger par la suite. 
De nombreux historiens, habitués aux réflexions subtiles et pleines de nuances de Bloch auront sans doute tendance à réagir avec impatience à ces remarques, et à les condamner pour leur abstraction excessive. Et il est vrai que Pike ne s'adressait pas à des historiens mais à des anthropologues et à des linguistes ${ }^{27}$. Pendant très longtemps, ces deux groupes ont eu affaire à cette distinction des niveaux émique et étique ; les historiens, au contraire, ont tout ignoré de cette distinction, à quelques exceptions près. (Moi-même je suis devenu conscient de cette différence émiquelétique il y a une vingtaine d'années, à savoir vingt ans après la publication du magnum opus de Pike ${ }^{28}$. Mais il ne serait peut-être pas sans pertinence d'essayer de traduire le passage de Pike que je viens de citer en utilisant des mots qui sont généralement associés à la recherche historique.

Le résultat serait à peu près le suivant :

"Les historiens commencent par des questions en utilisant des termes qui sont inévitablement anachroniques. Le processus de la recherche modifie les questions de départ sur la base des nouvelles sources, récupérant des réponses articulées dans le langage des acteurs et reliées aux catégories propres à leur société, qui est complètement différente de la nôtre ».

Ma traduction des « résultats provisoires " produits par la perspective étique - « Les historiens commencent par des questions en utilisant des termes qui sont inévitablement anachroniques » fait écho à une remarque de Bloch ${ }^{29}$. Des questions, et non pas des réponses : une distinction manquée par ceux qui ont insisté imprudemment sur le rôle de l'anachronisme dans la recherche historique ${ }^{30}$. On part de questions étiques en espérant obtenir des réponses émiques ${ }^{31}$.

27 "I am not a historical linguist/ Je ne fais pas de la linguistique historique " a pu écrire Pike ("On the Emics and Etics of Pike and Harris ", in Thomas N. Headland, Kenneth L. Pike, Marvin Harris (éd.), Emics and Etics. The Insider /Outsider Debate, Newbury Park, Sage Publications, 1990, p. 40.

28 "Saccheggi rituali. Premesse a una ricerca in corso ", séminaire bolognais coordonné par Carlo Ginzburg, Quaderni storici, 65 (1987), p. 615-636. Une exception significative est offerte par Simona Cerutti, "Microhistory: Social Relations versus Cultural Models? ", in Anna-Maija Castrén, Markku Lonkila, et Matti Peltonen (éd.), Between Sociology and History. Essays on Microhistory, Collective Action, and Nation-Building, p. 17-40 (voir mon commentaire, note 31 ).

29 «Les documents tendent à imposer leur nomenclature; l'historien, s'il les écoute, crit sous la dictée d'une époque chaque fois différente. Mais il pense, d'autre part, naturellement selon les catégories de son propre temps ", Marc Bloch, Apologie, op. cit., p. 959-960.

30 Nicole Loraux, "Éloge de l'anachronisme en histoire ", Le genre humain, 1993, "L'ancien et le nouveau ", p. 23-39 ; Jacques Rancière, «Le concept d'anachronisme et la vérité de l'historien ", L'inactuel, 6, 1996, p. 66-68 ; Georges Didi-Huberman, Devant le temps. Histoire de l'art et anachronisme des images, Paris, Les éditions de Minuit, 2000.

31 "Émique est une méthode d'analyse et non pas le contexte immédiat d'un comportement » écrit S. Cerutti en critiquant ma propre approche ("Microhistory », p. 35 ; c'est elle qui souligne). Pike qui évoque un "point de vue émique " - « an emic point of view » - et des unités 
On pourrait comparer mon essai de traduction avec l'une des règles du décalogue proposé il y a plusieurs années par Arnaldo Momigliano sous le titre «Les règles du jeu pour l'étude de l'histoire ancienne ». Ces règles s'appliquent à l'histoire de toutes les périodes :

Dès que nous pénétrons dans le champ de la recherche historique, Judaïsme, Chrétienté, Islam, Marx, Weber, Jung et Braudel nous apprennent à soumettre les sources à des questions spécifiques : elles n’affectent pas les réponses fournies par les sources. L'arbitraire de l'historien disparaît dès qu'il doit interpréter un document ${ }^{32}$.

Selon moi, entre le passage de Pike, la traduction que j'en propose et la règle de Momigliano, il n'y a pas de différences significatives. Les divergences sont ailleurs. Ce résidu étique qu'on ne peut annuler selon Pike, il nous faudrait le considérer de manière positive : comme un élément intrinsèque de l'activité de traduction qui est, selon l'étymologie, synonyme de l'interprétation. Il faut maintenir la tension entre nos questions et les réponses que nous obtenons de nos sources, quand bien même nos sources pourraient modifier nos questions de départ ${ }^{33}$. Si la différence entre nos mots et leurs mots est préservée avec soin, nous pourrons éviter de tomber dans deux sortes de pièges : l'empathie et la ventriloquie $^{34}$. Ces deux pièges sont reliés : en faisant fond sur la transparence des acteurs, nous leur attribuons notre langage et nos catégories. Il en résulte des distorsions insidieuses qui sont bien plus dangereuses (car plus difficiles à dénicher) que les énoncés grossièrement anachroniques du genre de l'homo oeconomicus et autres.

Le terme latin interpres nous rappelle que toute interprétation est une traduction et vice-versa. La question de la traduction apparaît dans les débats inspirés par les arguments de Pike. Un ensemble de réactions a été publié dans un livre intitule Emics and Etics: The Insider/Outsider Debate, qui part d'un colloque qui s'est déroulé à Phoenix en 1988. L'un des participants, Willard O. Quine, le philosophe rendu célèbre, entre autres, par ses réflexions sur la «traduction radicale ", achève ainsi son propos :

" émiques » n’aurait pas été d'accord avec elle. Mais la perspective émique ne peut être saisie qu'à travers la médiation d'une perspective étique : d'où la part active jouée par le chercheur (part que Cerutti considère comme arbitraire, ibid., p. 34), dans le processus de la recherche.

32 Arnaldo Momigliano, "Le regole del gioco nello studio della storia antica " [1974], Sui fondamenti della storia antica, Turin, Einaudi, 1984, p. 483 ; traduction française, "Règles du jeu pour étudier l'histoire antique » in Europe, 945-946, janvier-février 2008, p. 10-20.

33 Étrangement, la révision des questions de depart manque dans la version donnée par Clifford Geertz du cercle herméneutique : cf. " "From the Native's Point of View": On the Nature of Anthropological Understanding " [1974], in Local Knowledge. Further Essays in Interpretive Anthropology, New York, Basic Books, 1983, p. 55-70 ; traduction française, Savoir local, savoir global. Les lieux du savoir, Paris, PUF, 1986, 2012, p. 79-101.

34 Ce n'est qu'après avoir écrit ces pages que je me suis aperçu qu'on trouve la même métaphore in Lorraine Daston et Peter Galison, Objectivity, New York, Zone Books, 2007, p. 257 : « To ventriloquize nature " (mais le contexte tout entier est significatif). 
Et cependant il y a là, entre dedans et dehors, une asymétrie vitale. Notre engagement provisoire, et néanmoins responsable à l'égard de notre science s'étend à ce que nous disons au sujet des cultures exotiques, mais ne saurait s'étendre à ce que les indigènes disent de l'intérieur de ces cultures ${ }^{35}$.

L'asymétrie qui sépare nos mots de leurs mots, et que souligne Quine après Pike n'est pas étrangère aux historiens : comme le dit le dicton, « le passé est un pays étranger ${ }^{36}$. Certes, il n'est pas étonnant qu'une telle asymétrie ait été articulée et théorisée par un anthropologue. La distance, à la fois linguistique et culturelle, qui sépare les anthropologues de ceux qu'on appelle les " autochtones " empêche les premiers de se comporter comme les historiens le font souvent : à savoir comme s'ils étaient devenus les intimes des personnages avec lesquels ils ont affaire. Comme je l'ai indiqué auparavant, la ventriloquie est une maladie professionnelle dont sont souvent victimes les historiens. Mais pas tous, bien sûr.

On a pu parler d'une anthropologie émique, dont l'engagement spécifique consiste à sauver le "point de vue de l'autochtone ", comme Malinowski a pu le dire ${ }^{37}$. On pourrait parler, par analogie, d'une historiographie émique. Trois exemples splendides suffiront : les essais de Paul Oskar Kristeller et d'Augusto Campana sur les origines du mot " humaniste ", la conférence peu connue d'Ernst Gombrich sur la Renaissance comme période et comme mouvement ${ }^{38}$. Chacun de ces trois essais a pour but de reconstruire les catégories des acteurs en tant qu'elles se distinguent des catégories des observateurs - or ces catégories informent souvent la pensée d'un groupe qui s'étend bien au-delà $\mathrm{du}$ cercle des historiens professionnels. À la fin de son essai, Campana fait remarquer qu'on a pu parler récemment (le texte date de 1946) d'un "nouvel humanisme : et l'ancien mot a été chargé de nouveaux idéaux. Les philologues et les historiens du futur devront s'en occuper ".

Mais dans un postscriptum publié l'année suivante, Campana utilisait des mots plus forts : il croyait que Kristeller, dans l'essai qu'il avait écrit indépendamment sur le même sujet, avait démontré que le concept moderne

35 Willard V. Quine, "The Phoneme's Long Shadow ", in Etics and Emics, op. cit., p. 167.

36 David Lowenthal, The Past is a Foreign Country, Cambridge, Cambridge University Press, 1985.

37 Robert Feleppa, "Emic Analysis and the Limits of Cognitive Diversity ", in Etics and Emics, op. cit., p. 101 et suivantes

38 Paul O. Kristeller, "Humanism and Scholasticism in the Italian Renaissance " [1944-45], in Studies in Renaissance Thought and Letters, Rome, Edizioni di Storia e Letteratura, 1956, p. 553-583 (cf. aussi introduction, p. XI-XII) ; Augusto Campana, "The Origin of the Word "Humanist" " [1946], in Scritti, édition de Rino Avesani, Michele Feo, Enzo Pruccoli, I, Ricerche medievali e umanistiche, Rome, Edizioni di Storia e Letteratura, 2008, p. 263-28 ; Carlo Dionisotti, "Ancora humanista-umanista ", Scritti di storia della letteratura italiana, III (1972-1998), édition de Tania Basile, Vicenzo Fera et Susanna Villari, Rome, Edizioni di Storia e Letteratura, 2010, p. 365-370 ; Ernst H. Gombrich, "The Renaissance: Period or Movement " in Joseph B. Trapp (éd.) Background to the English Renaissance: Introductory Lectures, Londres, Gray-Mills Publishing, 1974, p. 9-30. 
"d'humanisme de la Renaissance [...] [était] intenable " ${ }^{39}$. Intenable, bien sûr, d'un point de vue philologique. Cela ne doit pas nous empêcher d'utiliser des catégories comme "Renaissance " (comme Campana lui-même a pu le faire par la suite $)^{40}$. Mais nous devons toujours être conscients que ces mots, pour utiles qu'ils puissent être, restent toujours conventionnels. Ceux qui font tant d'efforts pour découvrir les structures intrinsèques de l'humanisme, de la Renaissance, de la modernité, du XX $X^{e}$ siècle, ceux-là, pour le dire aimablement, perdent leur temps.

8. La dimension émique, que j'ai proposé, sous forme d'expérience, de trouver dans l'historiographie, peut être décrite en recourant à des mots plus anciens et plus familiers : la philologie, le savoir des antiquaires. (Et comme l'anthropologie est née du savoir des antiquaires, la boucle est bouclée). Mais une transposition mécanique de l'opposition entre émique et étique à l'intérieur $\mathrm{du}$ discours historiographique serait trompeuse. En s'appuyant sur leur propre pratique, les historiens pourraient faire remarquer que la distinction émiquel étique est plutôt simplificatrice. Comme le montre mon cas frioulan, les dimensions émique et étique sont elles aussi le théâtre de conflits : entre les inquisiteurs et les benandanti (dans le premier cas), entre des historiens d'orientations différentes (dans le dernier). Mais prendre conscience de la distinction émiquelétique pourrait aider les historiens à se libérer d'un travers ethnocentrique. Or cette tâche est devenue d'autant plus urgente dans un monde façonné par la globalisation - ce processus qui s'est développé sur plusieurs siècles mais qui a subi une accélération véritablement frénétique pendant les dernières décennies.

Les historiens doivent donc relever ce défi - la question devient : comment ? Un début de réponse se trouve dans les débats relatifs aux textes littéraires. L'essai d'Erich Auerbach "Philologie de la littérature mondiale [Weltliteratur] " peut servir de point de départ. Ce célèbre essai est paru en 1952 et il assume aujourd'hui une dimension presque prophétique ${ }^{41}$.

Une triste prophétie. En plein milieu de la Guerre Froide, Auerbach note une tendance répandue à l'homogénéité culturelle. En dépit des différences évidentes, ce phénomène frappait les deux blocs. Le monde devenait toujours plus semblable ; et même les états nations qui avaient été par le passé les agents

39 Augusto Campana, Scritti, p. 280-281.

40 Ibid., p. 405.

41 Erich Auerbach, "Philologie der Weltliteratur", in Gesammelte Aufsätze zur romanischen Philologie, Bern, Francke, 1962, p. 301-310. Cf. aussi l'introduction de Enrica Salvaneschi et Silvio Endrighi ("La letteratura cosmopolita di Erich Auerbach »), à Philologie der Weltliteratur - Filologia della letteratura mondiale, traduction de Regina Engelmann, Castel Maggiore (Bo), Book, 2006 ; cf. "La philologie de la littérature mondiale ", traduit de l'allemand par Diane Meur et présenté par Christophe Pradeau in Christophe Pradeau et Tiphaine Samoyault (éd.), Où est la littérature mondiale?, Saint-Denis, PUV, 2005, p. 15-37. 
de la différenciation culturelle perdaient une bonne partie de leur pouvoir. La culture de masse (un terme qu'Auerbach n'utilise pas, mais c'était là l'essentiel de son analyse) se propageait sur toute la surface du globe. Une Weltliteratur émergeait dans un contexte complètement différent de celui qu'avait pu imaginer Goethe : dans cette littérature mondiale l'Europe jouait un rôle marginal. Face à son expansion considérable dans l'espace et dans le temps, même un savant aux vues aussi larges que l'était Auerbach, mesurait bien le caractère inadéquat de ses instruments. Il donnait ainsi quelques conseils aux jeunes chercheurs, les uns positifs, les autres négatifs. D’une part, il leur suggérait d'éviter à la fois le recours à des concepts généraux comme Renaissance ou Baroque et les études monographiques basées sur les œuvres d'un seul auteur. Et d'autre part, il leur recommandait de trouver des détails spécifiques qui puissent leur servir de points d'articulation (Ansatzpunkte).

Auerbach faisait allusion à la méthode qui avait inspiré son grand livre, Mimésis. Mais en 1952 les réflexions qu'il avait avancées moins d'une décennie auparavant dans la dernière section de Mimésis, se développaient dans une toute autre direction. S'il n'est plus possible de tabler sur la tradition littéraire européenne, l'enjeu de la généralisation prend le devant de la scène, fût-ce de manière implicite. Généralisation - soit, mais à partir d'où et dans quel but ?

Il y a quelques années, dans un essai intitulé « Conjectures sur la littérature mondiale " (qui, curieusement, ne mentionne pas Auerbach) Franco Moretti s'est attaqué avec courage à ces questions ${ }^{42}$. Face au défi que représente une quantité aussi considérable de textes qu’aucun chercheur en littérature comparée ne pourra jamais maitriser, Moretti suggère une solution drastique : la lecture de seconde main. Les chercheurs engagés dans une approche comparée de la littérature seront en mesure de poser des questions générales en s'appropriant les vues des chercheurs qui les ont précédés et ont travaillé dans une perspective plus circonscrite, centrée sur une littérature nationale spécifique. De la sorte, l'étude comparée de la littérature ne se devrait plus se fonder sur une lecture de près mais sur une lecture de loin. Cette proposition, avancée de manière délibérément provocatrice, se trouvait encadrée par un argument qui se fonde sur l'essai de Marc Bloch duquel je suis parti : « Pour une histoire comparée des sociétés européennes ». Une comparaison des deux passages significatifs (d'abord le texte de Moretti, puis celui de Bloch) pourrait s'avérer utile. Moretti d'abord :

Selon une formule délicieuse que Marc Bloch qualifia lui-même de "slogan », on peut résumer le travail de l'histoire sociale comparée en ces termes : « des années d'analyse pour un jour de synthèse "; le lecteur de Braudel ou de Wallerstein voit immédiatement ce que Bloch veut dire. Dans l'œuvre de Wallerstein, le texte qui lui revient en propre, son "jour de synthèse ", occupe un tiers de la

42 Franco Moretti, «Conjectures on World Literature ", New Left Review, n. s. 1, 2000, p. 54-68. Jonathan Arac, "Anglo-Globalism? ", New Left Review, 16, 2002, p. 35-45, suggère un rapprochement entre les essais de Moretti et ceux d'Auerbach. 
page, un quart, la moitié de la page peut-être, pour le reste, il s'agit de citations (mille quatre cents dans le premier volume de The Modern World-System - Le système monde moderne). Des années d'analyse, d'analyse menée par d'autres dont la page de Wallerstein offre la synthèse sous une forme systématique ${ }^{43}$.

"C'est toujours le vieux mot: des années d'analyse pour une journée de synthèse » : telle est la formulation de Bloch. Il renvoyait à un passage de l'introduction de Fustel de Coulanges à La Gaule romaine, publiée en 1875. Dans une note de bas de page, Bloch donnait la citation précise : "Exactement : "pour un jour de synthèse, il faut des années d'analyse" ". Mais la répétition de la formule de Fustel de Coulange a bien peu d'importance comparée au commentaire qu'en offre Bloch :

Mais on cite trop souvent cette maxime, sans y ajouter le correctif nécessaire :

l' " analyse " ne sera utilisable pour la "synthèse " que si, dès le principe, elle l'a

en vue et se préoccupe de la servir ${ }^{44}$.

La remarque de Bloch va dans la direction opposée à la lecture de Moretti ${ }^{45}$. Il ne s'agit pas, comme le pensent les positivistes, d'accumuler les briques, c'est-à-dire les recherches monographiques, pour édifier une construction qui existerai seulement dans la tête de l'architecte (ou du professeur de littérature comparée). Il s'agit bien plutôt de rassembler des témoignages selon un programme qui indique déjà une approche synthétique. En d'autres termes, il s'agit d'identifier des cas, qui pourront mener à des généralisations. Mais dès lors que la plupart des sources ont été rassemblées, filtrées ou traitées par des chercheurs qui nous précédent, et qui sont partis de questions différentes des nôtres, l'histoire de l'historiographie doit être incorporée dans la recherche historique elle-même. Plus nous sommes loin des sources, plus le risque de se laisser capturer par des hypothèses avancées par des intermédiaires ou par nous-mêmes devient grand. En d'autres termes, nous courons le risque de trouver ce que nous cherchons - et rien d'autre.

Cette lecture déformée du texte de Bloch est d'autant plus surprenante que Moretti lui-même, dans un essai brillant, publié avec ses « Conjectures sur la littérature mondiale ", montre que la seule manière de relever le défi lancé par cette masse énorme et impossible à maîtriser de textes publiés ou oubliés, est de travailler sur une étude de cas : une analyse menée en première main, d'une série limitée de textes, identifiés au moyen d'une question spécifique. Ce second essai, intitulé "The Slaughterhouse of Literature [L'abattoir de la littérature] » (une allusion à un aphorisme de Hegel), traite d'un procédé littéraire que Conan Doyle a placé, de manière presque involontaire, au cœur de

43 Franco Moretti, «Conjectures on World Literature », art. cit., p. 56-57.

44 Marc Bloch, "Pour une histoire ", art. cit., p. 38.

45 Le texte de Marc Bloch est cité de première main (sans la remarque qui le suit immédiatement) in Franco Moretti, Il romanzo di formazione, Turin, Einaudi, 1999, p. XX ; « Prefazione 1999 ». 
ses histoires de détective : «les traces " ${ }^{46}$. Il y a plusieurs années j’ai moi-même écrit un essai intitulé "Traces » qui traite de Sherlock Holmes et d'autres questions dans une perspective différente ${ }^{47}$.

Si je ne m'abuse, l'essai de Franco Moretti et le mien recourent au procédé connu comme mise en abyme : les traces, comme objet d'étude, se trouvent étudiées par les moyens d'une approche qui se fonde elle-même sur des traces : les détails reflètent l'ensemble ${ }^{48}$. Mais les traces nécessitent une lecture de première main : la personne responsable de la synthèse finale ne peut déléguer cette tâche à d'autres. Plus encore, une lecture analytique est compatible avec une énorme quantité de sources. Les personnes qui ont l'habitude de mener des recherches dans les archives savent qu'on peut passer beaucoup de temps à feuilleter d'innombrables dossiers et à inspecter le contenu de boîtes sans nombre avant d'être soudainement arrêté par un document qui nécessitera des années d'analyse. De la même manière, un poulet (j'espère que personne ne sera scandalisé par une telle comparaison) ne cesse d'aller et venir en jetant son regard tout autour de lui, avant de se saisir brutalement du vers caché jusque-là dans le sol de la basse-cour. Nous voici de nouveau ramenés aux Ansatzpunkte : les points spécifiques qui pourront fournir, selon Auerbach, les semences d'un programme de recherches détaillé doté d'un réel potentiel de généralisation - en d'autres termes, des cas. Les cas exceptionnels sont particulièrement prometteurs puisque les anomalies, comme il est arrivé à Kierkegaard de le remarquer, sont plus riches, d'un point de vue cognitif, que les normes dans la mesure où les premiers incluent invariablement les secondes, alors que l'inverse n'est pas vrai ${ }^{49}$.

9. Pendant un certain nombre d'années, les cas ont fait l'objet d'une attention croissante, en partie reliée aux débats qui couraient sur la microhistoire : un terme dont le préfixe - micro - fait allusion, comme on l'a souligné de nombreuses fois (mais pas assez peut-être) au microscope, au regard analytique, et non pas aux dimensions, supposées ou réelles, de l'objet soumis à l'analyse ${ }^{50}$.

46 Franco Moretti, "The Slaughterhouse of Literature ", Modern Language Quarterly, 61, 2000, p. $207-227$.

47 Carlo Ginzburg, "Spie: radici di un paradigma indiziario " (1979), in Miti emblemi spie. Morfologia e storia, Turin, Einaudi, 1986, p. 158-209 ; «Traces : racines d'un paradigme indiciaire " in Mythes emblèmes traces, Lagrasse, Verdier, 2010, p. 218-294.

48 Lucien Dällenbach, Le récit spéculaire : essai sur la mise en abyme, Paris, Seuil, 1977.

49 Cf. Carl Schmitt, Politische Theologie. Vier Kapitel zur Lehre von der Souveränität, Zw. Ausgabe, München \& Leipzig: Dunckler \& Humblot, 1934, p. 33. Schmitt se réfère à un « théologien protestant " qu'il ne nomme pas. Je remercie Henrique Espada Lima qui m’a rendu conscient de la provenance de cette remarque que je m'étais appropriée sans le savoir.

50 La meilleure introduction au sujet reste le chapitre «Kasus » in André Jolles, Einfache Formen, Halle, 1930, p. 171-199 ; Formes simples, Paris, Le Seuil, 1972. Cf. aussi John Forrester, "If p, then what? Thinking in cases ", History of the Human Sciences, 9, 1996, p. 1-25, et JeanClaude Passeron \& Jacques Revel (éd.), Penser par cas, Paris, 2005. 
Et pourtant la microhistoire, qui se fonde sur la recherche analytique (et donc sur la recherche de première main) vise la généralisation : un mot qu'on a la mauvaise habitude de considérer comme allant de soi. Des réflexions supplémentaires sont pourtant nécessaires pour explorer la vaste gamme de ses variétés, fondées sur des points de départs différents (questions et réponses), sur des types d'analogies différentes (métonymie, métaphore) et ainsi de suite ${ }^{51}$.

On pourrait objecter qu'il n'y a pas de place pour la microhistoire dans un monde globalisé. Pour moi, c'est le contraire qui est vrai. La réception internationale de la microhistoire peut être facilement interprétée dans une perspective géopolitique. La première vague d'intérêt pour la microhistoire, après sa naissance en Italie, s'est manifestée en Allemagne, en France, en Angleterre et aux États-Unis. Elle a été suivie par une seconde vague, liée aux périphéries ou demi-périphéries : la Finlande, la Corée du Sud, l'Islande ${ }^{52}$. La microhistoire a permis de subvertir des hiérarchies préexistantes grâce la pertinence intrinsèque - démontrée a posteriori - de l'objet soumis à l'examen. Cela n'a pas grand-chose à voir avec ce qu'on a pu appeler "l'Anglobalisation": le mouvement impérialiste (au délà de ses intentions) qui a porté à des études de littérature comparée écrites en anglais, fondées sur des études écrites pour la plupart en anglais, portant sur des textes littéraires la plupart du temps écrits dans des langues qui n'étaient pas l'anglais ${ }^{53}$.

S’appuyer sur la microhistoire pour subvertir les hiérarchies politiques et historiographiques remonte à un passé lointain. Ce n'est pas la tribu X qui compte, a dit un jour Malinowski, ce sont les questions que l'on pose à la tribu X. C'est dans un esprit analogue que Marc Bloch a soutenu que l'histoire locale doit

51 Roman Jakobson, " Deux aspects du langage et deux types d'aphasie ", in R. Jakobson, Essais de linguistique générale, t. I, Paris, Minuit, 1963. On peut compter sur l'aide consistante du livre d'Enzo Melandri La linea e il circolo. Studio logico-filosofico sull'analogia [1968], $2^{\text {de }}$ édition, avec une introduction de Giorgio Agamben, annexes de Stefano Besoli et Renzo Brigati, Macerata, Quodlibet, 2004.

52 Quelques références bibliographiques : Edwin Muir et Guido Ruggiero (éd.), Microhistory and the Lost People of Europe, Baltimore, Johns Hopkins University Press, 1991 ; Matti Peltonen, "Carlo Ginzburg and the new microhistory "Suomen Antropologi, I (1995), p. 2-11 ; Jacques Revel (éd.), Jeux d'échelles : la micro-analyse à l'expérience, Paris, Gallimard-Seuil, 1996 ; Kwak Chasob (éd.), Mishisa ran muoshinga, Séoul, Purun Yoksa, 2000 ; Carlo Ginzburg, David Ólafsson, Sygurdur G. Magnússon, Molar og mygla. Um einsögu og glataðan tíma, Reykiavík, Bjartur-ReykjavíkurAkademían, 2000 ; Matti Peltonen, "Clues, Margins, and Monads: The Micro-Macro Link in Historical Research ", History and Theory, 40/3, 2001, p. 347-359 ; Sygurdur G. Magnússon, «The Singularization of History: Social history and Microhistory within the Postmodern State of Knowledge ", Journal of Social History, 36, 2003, p. 701-735.

53 La critique a été formulée par Jonathan Arac, "Anglo-Globalism? ", New Left Review, 16, 2002, p. 35-45. Dans sa réponse, Moretti ne tient pas compte de ce problème, cf. "More Conjectures ", New Left Review, 20, 2003, p. 73-81 (la note 8 traite du langage utilisé par les critiques et non l'approche de deuxième ou de troisième main des textes traduits qu'étudieraient les métacritiques qui travailleraient dans une perspective comparée). 
être étudiée à partir de questions qui comportent des implications générales. À la lueur de ce que j'ai dit jusque-là, la convergence entre l'anthropologie et l'histoire doit apparaître comme une évidence. Dans un monde comme le nôtre, dans lequel quelques historiens, réagissant à la pseudo-universalité de l'Homo religiosus de Mircea Eliade, ont souligné la dimension ethnocentrique, romaine et chrétienne du mot "religion", les études de cas, reliées à des contextes spécifiques, semblent prometteuses, dans la mesure où elles permettent de nouvelles généralisations, engendrant de nouvelles questions et de nouvelles recherches ${ }^{54}$. Des réponses émiques font naître des questions étiques et vice versa.

Je n'ai pas l'intention d'achever mon propos en chantant les louanges de la microhistoire. Les étiquettes ne m'intéressent pas ; la mauvaise microhistoire est de la mauvaise histoire. Aucune méthode ne peut nous garder de nos limites et de nos erreurs. Quand nous nous adressons aux générations qui viennent, nous devons admettre franchement nos défauts, en décrivant ce que nous avons essayé de faire contre vents et marées. Les générations qui viennent nous écouteront et elles feront autre chose que nous, comme ce fut toujours le cas. "Tristo è lo discepolo che non avanza il suo maestro" (l'élève qui ne dépasse pas son maître est un mauvais élève) a dit un jour Léonard.

\author{
Carlo Ginzburg \\ UCLA / Scuola Normale Superiore, Pisa \\ Traduction de Martin Rueff
}

\begin{abstract}
Résumé
L'une des questions essentielles qui se pose aujourd'hui dans le travail de l'historien est le choix de catégories appropriées à redonner la parole aux acteurs du passé. À la différence des anthropologues, habitués à la distance, à la fois linguistique et culturelle, qui les sépare de leurs objets d'étude, les historiens en faisant fond sur la transparence des acteurs, sont enclins à leur attribuer leur langage et leurs catégories. Cet article s'inscrit dans le débat actuel sur le rôle de l'anachronisme dans la recherche historique ; à l'aide d'outils élaborés dans les années 1960 par le linguiste américain Kenneth L. Pike, il vise à accentuer la distinction entre deux niveaux d'analyse de la réalité, celui de l'observateur et celui de l'acteur, baptisés respectivement niveau étique (de phonétique) et niveau émique (de phonémique). Le but de cette opération de mise à distance entre nos mots et les leurs est la possibilité de récupérer des fragments " émiques » à même de dialoguer avec nos catégories " étiques » et, en fin des comptes, de les reformuler.
\end{abstract}

\title{
Mots-clés
}

Historiographie, anachronisme, Kenneth L. Pike, étique/émique.

54 Arnaldo Momigliano, "Questioni di metodologia della storia delle religioni », in Ottavo contributo alla storia degli studi classici e del mondo antico, Rome, Edizioni di Storia e Letteratura, 1987, p. 402-407 ; Jonathan K. Smith, Relating Religion: Essays in the Study of Religion, Chicago: Chicago University Press, 2004. 


\begin{abstract}
Among the essential questions being asked concerning the work of historians today is the choice of categories suited to grant speech anew to actors from the past. Unlike anthropologists, used to distance, both linguistic and cultural, which separates them from their objects of study, historians in laying their foundation upon transparency of the actors, are inclined to attribute to them their own language and their own categories. This article enters into the current debate of the role of anachronism in historical research; using tools elaborated during the 1960's by the American linguist Kenneth L. Pike, it seeks to accentuate the distinction between two levels of the analysis of reality/reality analysis, that of the observer and that of the actor, respectively baptized as the "etic" level (of phonetic) and the "emic" level (of phonemic). The goal of this operation of distancing our words from theirs is the possibility of recuperating fragments "emics" as well as to dialogue with our "etic" categories and, finally, to reformulate them.
\end{abstract}

\title{
Keywords
}

Historiography, anachronism, Kenneth L. Pike, eticlemic. 